The future of cancer chemotherapy lies in increasing the specificity of treatment and diminishing its toxicity. The lack of specific treatment for many cancers emphasises the importance of the clinical trial in assessing treatment: this will jettison irrelevant or harmful measures, preserving only those of proved value. We must now put similar efforts into identifying those factors, including antiemetics, that can best reduce the one consequence of treatment most often raising doubts in the patient's mind about whether the cure is really worth the discomfort of treatment, however temporary this may be.

1 Morran, C, et al, British Medical fournal, 1979, 1, 1323.

${ }^{2}$ Moertel, C G, and Reitemeier, R J, Gastroenterology, 1969, 57, 262.

${ }^{3}$ Sallan, S E, Zinberg, N E, and Frei, E, New England fournal of Medicine, 1975, 293, 795 .

4 Weil, A T, Zinberg, N E, and Nelsen, J M, Science, 1968, 162, 1234.

${ }^{5}$ McCarthy, L E, and Borison, H L, The Pharmacologist, 1977, 19, 230.

${ }^{6}$ Herman, T S, et al, Biomedicine, 1977, 27, 331.

${ }^{7}$ Herman, T S, et al, New England fournal of Medicine, 1979, 300, 1295.

\section{Tardive dyskinesia}

If anyone doubts the continuing truth of Voltaire's claim that doctors pour drugs of which they know little into patients of whom they know even less, the phenomenon of tardive dyskinesia should convince them. Though it was first reported ${ }^{1}$ in 1957, for many years psychiatrists were reluctant to accept that tardive dyskinesia was a side effect-all too often an enduring one-of the neuroleptic drugs ${ }^{2} 3$ that are their principal weapons against schizophrenia. The syndrome is chiefly characterised by repetitive pouting of the lips and protrusion of the tongue, often accompanied by bizarre facial grimacing and sometimes more widespread choreiform movements. Gibson ${ }^{4}$ found that in chronic schizophrenic patients receiving depot neuroleptics the syndrome appeared with steadily increasing frequency and had affected about a quarter of them after three years. Nor is it only schizophrenic patients who may be affected. Paulson ${ }^{2}$ reported 14 sufferers who were neither institutionalised nor psychotic but who had all received neuroleptics, some of them for many years.

When many different treatments are recommended for a condition usually none of them is particularly effective. This is certainly the case with tardive dyskinesia. For example, though a recent well-controlled trial ${ }^{5}$ showed that muscimol (a structural analogue of $\gamma$-aminobutyric acid, GABA) improved symptoms in tardive dyskinesia unfortunately it also caused an increase in psychotic symptoms.

Because treatment is so unsatisfactory attempts have been made to detect patients who may be especially likely to develop tardive dyskinesia. Those with brain damage and the elderly (not necessarily the same people) are known to be vulnerable. Wegner $e t a l^{6}$ have recently reported the presence of a characteristic pattern in the electroencephalogram in $95 \%$ of patients with tardive dyskinesia, whereas it is found in only $33 \%$ of controls; but whether this finding is a precursor or a result of tardive dyskinesia is not yet known.

In our present state of knowledge, the best hope of prevention of tardive dyskinesia remains care and discrimination in the use of neuroleptics. Not all schizophrenic patients require medication, and in those who do short "drug holidays" may reduce the likelihood of tardive dyskinesia-though there is no proof as yet. Most reports suggest that routine anticholinergic medication increases the risk of tardive dyskinesia, though Gibson ${ }^{4}$ disagrees. Clearly, neuroleptics should be prescribed for patients with conditions other than schizophrenia only after most careful consideration of the alternatives, especially where long-term use is likely. Early diagnosis of tardive dyskinesia may be important in arresting its progress, but it may also be difficult in a condition which, perhaps surprisingly, often seems to trouble the patients less than it troubles their physicians. ${ }^{2}$ In established cases, a gradual reduction or withdrawal of neuroleptic drugs may be helpful, though this sometimes leads to an initial worsening of the dyskinesia. Such a policy may not be practicable if it results in a recurrence of the underlying psychiatric disorder. Paradoxically, increasing the dosage may improve matters in some cases. Changing to a different neuroleptic may be worth a try. If that fails, all that remains is treatment with one of the many drugs that have been claimed to relieve symptoms; these include deanol, diazepam, baclofen, alphamethyltyrosine, tetrabenazine, and reserpine.

In the long term the answer must lie in the development of a new class of neuroleptic drugs that will control schizophrenia without producing tardive dyskinesia. A report by Shopsin et $a l^{7}$ indicates that clozapine-a dibenzazepine with some important pharmacological differences from most of the standard neuroleptics-may be such a compound, but psychiatry has seen so many false dawns that it would be premature to cheer. The ghost of Voltaire is there to remind us that, even if further trials confirm the safety of clozapine in respect of tardive dyskinesia, it may turn out to produce other, as yet unknown side effects in long-term use.

\footnotetext{
${ }^{1}$ Schonecker, M, Nervenarzt, 1957, 28, 35.

2 Paulson, G W, New York State Fournal of Medicine, 1979, 79, 193.

${ }^{3}$ Lancet, 1979, 2, 447.

4 Gibson, A C, British fournal of Psychiatry, 1978, 133, 361.

5 Tamminga, C A, Crayton, J W, and Chase, T N, Archives of General Psychiatry, 1979, 36, 595.

6 Wegner, J T, et al, Archives of General Psychiatry, 1979, 36, 599.

7 Shopsin, B, et al, Archives of General Psychiatry, 1979, 36, 657.
}

\section{Systemic sclerosis in old age}

Very elderly patients are referred for specialist opinions relatively rarely. Because of this bias, many diseases have been mistakenly thought to be rare in old age. In systemic sclerosis early reports suggested that it was predominantly a disease of middle life and a comparative rarity in old age. ${ }^{1-3}$ Before too long, however, the highest incidence was reported ${ }^{4}$ to be in patients aged over 65 . This revised view was confirmed in a personal series of 15 cases seen by one physician in geriatric medicine in 11 years ${ }^{5}$-representing an incidence of about one per 1000 elderly patients admitted under his care. A recent paper from a department of geriatric medicine ${ }^{6}$ has now drawn attention to the ease with which the diagnosis of systemic sclerosis may be overlooked in the elderly because of the minor nature of the skin changes.

What, then, is the clinical picture of systemic sclerosis in old age reported in these two papers? Most strikingly, all the patients were women. Their average age was 80 , closely corresponding to the overall average for women admitted to a geriatric department. All had Raynaud's phenomenon affecting the hands, and it was this symptom - otherwise rare in old agewhich commonly drew attention to the disease. All the patients had skin changes in their hands, but these were most often not striking. Nevertheless, more than half had evidence of old or recent skin ulceration, whitlows, or necrosis of the pulp. 